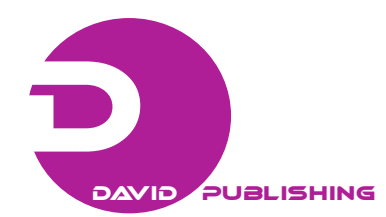

\title{
Rheological and Morphological Properties of Composites Based on Polylactide and Talc
}

Talita F. Cipriano ${ }^{1}$, Ana Lúcia Nazareth da Silva ${ }^{1}$, Antonio Henrique Monteiro da Fonseca Thomé da Silva ${ }^{2}$, Ana Maria Furtado de Sousa ${ }^{3}$, Gustavo Monteiro da Silva ${ }^{3}$ and Christine Rabello do Nascimento ${ }^{4}$

1. Instituto de Macromoléculas Professora Eloisa Mano (IMA/UFRJ), Rio de Janeiro 21945-970, Brasil

2. Departamento Agrícola e Meio Ambiente (UFF), Niterói 24210-240, Brasil

3. Instituto de Química (IQ/UERJ), 20550-013, Brasil

4. Programa de Engenharia Metalúrgica (PEMM/COPPE/UFRJ), Rio de Janeiro 21941-908, Brasil

Received: November 02, 2013 / Accepted: November 20, 2013 / Published: November 25, 2013.

\begin{abstract}
Polylactide (PLA) is an emerging material mainly because it can be synthesized from renewable resources and is thus environmentally and ecologically safe. In this study, composites of PLA and talc were prepared by melt-blending technique, using a twin screw extruder. The influence of talc content on the rheological properties of pure PLA and its composites were evaluated. Dynamic rheological tests show that the incorporation of talc particles into PLA decreased system viscosity due to the filler's lubricant effect. However, as talc content increased, system's viscosity tended also to increase and materials with higher elastic behavior were produced, further with a higher plastic behavior in relation to pure PLA. This behavior can be observed by SEM micrographs.
\end{abstract}

Key words: PLA, talc, composite, morphology, rheological properties.

\section{Introduction}

Biodegradable polymers have been attracted great interest from both academic research and industrial manufacturing due to a growing environmental consciousness among consumers, manufactures and governments [1]. Polylactide (PLA) is highly important today as a biodegradable and bio-based polymer. It has been widely founded acceptance in application areas such as food packaging, bags, agricultural film and many others market applications [2, 3]. Due to it, PLA can be a good alternative to traditionally plastic, such as polyethylene and polypropylene [4, 5].

PLA is produced via fermentation or chemical synthesis from a bio-derived monomer, lactic acid

\footnotetext{
Corresponding author: Ana Lúcia Nazareth da Silva, D.Sc., research field: polymeric materials. E-mail: ananazareth@ima.ufrj.br.
}

(2-hydroxy propionic acid) and it can present a variable molecular weight range [6].

High molecular weight PLA can be processed into fibers, and articles with rigidity and strength, which are useful at commodity scale, with good optical, physical, mechanical, and barrier properties [3].

However, commercialized PLA has low crystallinity and a slow rate of crystallization, which limits its use in plastic or film application. In general, the addition of a nucleating agent to polymers with a medium crystal growth rate has been widely practiced in the fabrication of plastics, not only for improving the mechanical properties, but also for shortening the cycle period in injection molding processes. Conventional nucleating agents, such as talc, are effective for PLA [7].

It is known that the viscoelastic properties of filler-containing polymer composites have both basic 
and practical importance since they are related to composite processing behavior and morphology [8, 9].

With the purpose of increasing the understanding of the influence of particulate fillers on the PLA properties, PLA/talc composites with different loading of talc are made by melt blending in this report. Considering the dispersion of the filler in matrix is important to the final properties, such as rheological behavior, a study illustrating the structure-property relationship was done, by investigating the morphology and dynamic rheological properties of the PLA and PLA/talc composites.

\section{Materials and Methods}

\subsection{Materials}

The commercial PLA pellets with grade name INGEO 2003D were supplied by Natureworks LLC and the commercial mineral filler, talc, was donated by Magnesita Company. Tables 1 and 2 show the characterization of the PLA and the physical properties of talc, the mineral filler used as nucleating agent, respectively.

Table 1 PLA characterization.

\begin{tabular}{lll}
\hline Physical and Mechanical Properties & $\begin{array}{l}\text { INGEO } \\
\text { 2003D }\end{array}$ & $\begin{array}{l}\text { ASTM } \\
\text { Method }\end{array}$ \\
\hline Specific gravity & 1.24 & D792 \\
MFI, g/10 min $\left(210^{\circ} \mathrm{C}, 2.16 \mathrm{~kg}\right)$ & 6 & $\mathrm{D} 1238$ \\
Tensile strength at break (MPa) & 53 & D882 \\
Tensile yield strength (MPa) & 60 & D882 \\
Tensile modulus (GPa) & 3.5 & D882 \\
Notched Izod impact (J/m) & 16 & D256 \\
$\mathrm{Mn}^{*}(\mathrm{~g} / \mathrm{mol})$ & 114,317 & - \\
$\mathrm{Mw}^{*}(\mathrm{~g} / \mathrm{mol})$ & 181,744 & - \\
$\mathrm{DMM}^{*}$ & 1.59 & - \\
\hline
\end{tabular}

*GPC measurements in a Viscotek equipment.

Table 2 Physical characteristics of talc.

\begin{tabular}{ll}
\hline \multicolumn{3}{c}{ Physical properties } \\
\hline BET Surface Area $\left(\mathrm{m}^{2} / \mathrm{g}\right)^{\mathrm{a}}$ & 5.97 \\
Volume of pores $\left(\mathrm{cm}^{3} / \mathrm{g}\right)^{\mathrm{a}}$ & 0.0203 \\
Average pore diameter $(\AA)^{\mathrm{a}}$ & 45.55 \\
Particle diameter, D-50 $(\mu \mathrm{m})^{\mathrm{b}}$ & 11.58 \\
\hline a Measured in IMA laboratory & using ASAP \\
equipment. b Measured in CETEM laboratory using MALLS, \\
Hydro 2000 SM equipment.
\end{tabular}

Fig. 1 shows the micrographs of the talc particles obtained by using a Jeol scanning electron microscope (SEM), model JSM 5610 LV, from IMA laboratory.

\subsection{Sample Preparation}

PLA and talc were mixed in a plastic bag before being extruded in a twin-crew co-rotating extruder (TeckTril, model DCT-20). The screw diameter was $20 \mathrm{~mm}$ with $\mathrm{L} / \mathrm{D}=36$. The temperature profile was $45 / 210 / 210 / 220 / 220 / 210 / 200 / 200 / 200 / 190{ }^{\circ} \mathrm{C}$. The screw speed was set at $200 \mathrm{rpm}$. The extrudates of specimens were pelletized and then dried in a vacuum oven during night at $60^{\circ} \mathrm{C}$.

Table 3 shows the material compositions prepared in the extruder.

\subsection{Rheological Analysis}

The viscosities of the polymers were measured in the low shear rate range ( $\omega=0.01-600 \mathrm{rad} / \mathrm{s}$ ) on a plate-plate type rheometer (AR 2000, TA Instruments) at $190{ }^{\circ} \mathrm{C}$. A strain sweep test was initially conducted to determine the linear viscoelastic region of the materials. Dynamic frequency sweep test (strain: 5\%; frequency: 0.01 to $600 \mathrm{rad} / \mathrm{s}$ and temperature at $190{ }^{\circ} \mathrm{C}$ ) were subsequently performed to determine the dynamic properties of the materials, using a parallel-plate geometry.

The composite melt flow index (MFI) was analyzed in order to evaluate the effect of the nucleating agent on the flow behavior of the materials. The analysis was determined on a LMI-4003, Dynisco, according to ASTM D1238.

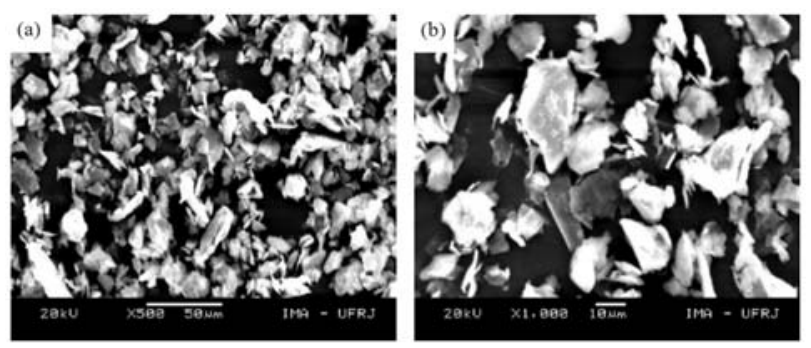

Fig. 1 SEM micrographs of talc particles (a) 500×; (b) 1,000×). 
Table 3 Sample compositions of PLA/nucleating agent.

\begin{tabular}{ll}
\hline Sample codes & Composition PLA/Talc (wt.\%) \\
\hline A & $100 / 0$ \\
B & $97 / 3$ \\
C & $95 / 5$ \\
D & $93 / 7$ \\
\hline
\end{tabular}

\subsection{Morphological Analysis}

A Scanning electron microscope (JSM 5610 LV, Jeol Ltd) was used to record the morphologies from fracture surface samples. The dispersion of the filler on the PLA matrix was observed at an accelerating voltage of $25 \mathrm{kV}$. Before examination all fracture surfaces were coated with gold.

\section{Results and Discussion}

\subsection{Rheological Analysis}

Fig. 2 shows the variation of the magnitude of the complex viscosity $\left(\eta^{*}\right)$, with the investigated frequencies for PLA and PLA/talc composites, with 3, 5 and 7 wt.\% of the mineral filler.

It can be observed that PLA presents a higher viscosity in the frequency range analyzed, as talc is added to the polymer matrix (regardless the amount of it), a decrease in viscosity values was observed compared to pure PLA. This behavior can be related to the lubricant effect of the mineral filler on the PLA chains. However, as the talc content increased, an increasing in the viscosity values was observed. This behavior might be associated with restriction of chain mobility caused by the reinforcing effect of the filler on the PLA matrix.

The frequency at which the G' and G” moduli curves cross reflect the transition from viscous to elastic response of the viscoelastic melts [10].

Fig. 3 shows the variation of the cross point (G'/G”) in relation to the polymer molecular structure, where G' is the storage modulus and G” is the loss modulus.

Table 4 shows the cross point values and the frequencies where these cross points occurred. The table also shows de weight average molecular weight $(\mathrm{Mw})$ determined from TA Data Analysis software.
MFI data is also presented in this table.

Table 4 shows that as talc was added to PLA matrix, a decreasing in the cross point (G'/G”) and an increasing in the crossover frequency values were observed. It indicates that materials with more viscous behavior (lower viscosity) were produced when talc was added. These results can be confirmed with $M_{w}$ and MFI values.

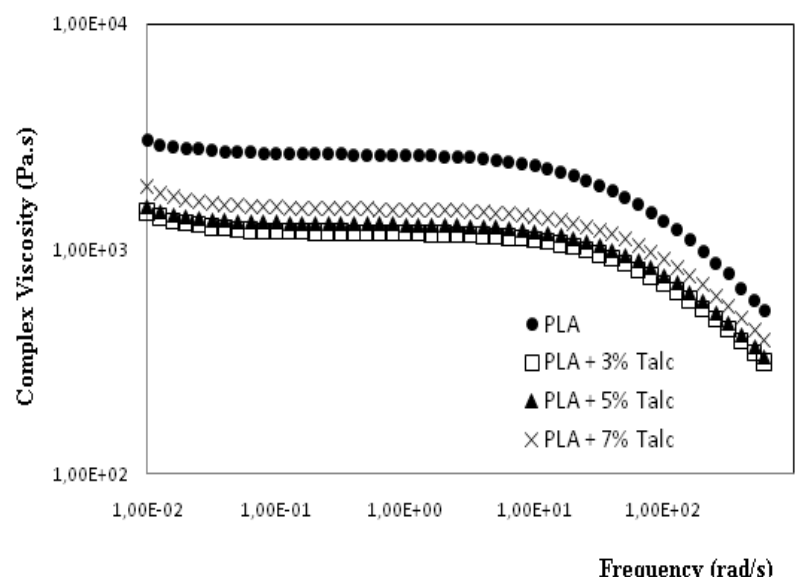

Fig. 2 Complex viscosity $\left(\eta^{*}\right)$ versus frequency for PLA and PLA/talc composites.

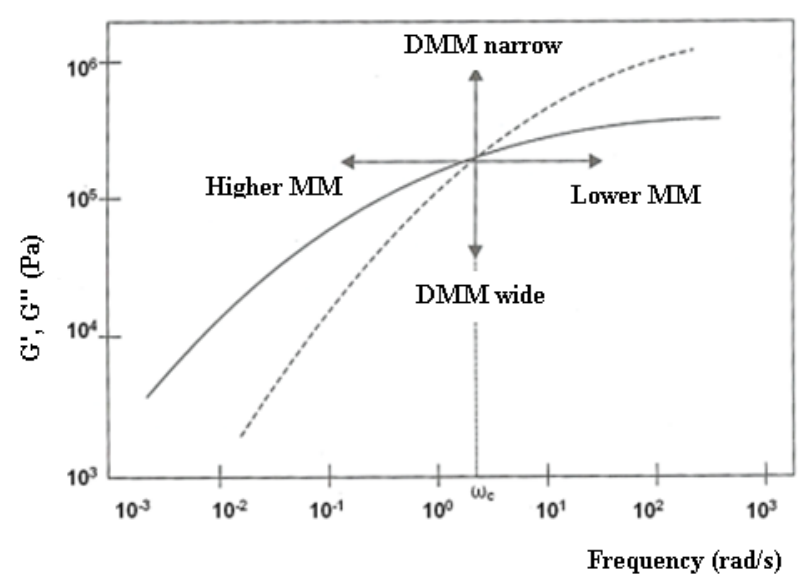

Fig. 3 Storage shear modulus (G') and loss modulus (G”) versus frequency $(\mathrm{MM}=$ molar mass and $\mathrm{DMM}=$ molar mass distribution) [11].

Table 4 Modulus and frequency values in the cross point G'/G” for the compositions analyzed.

\begin{tabular}{lllll}
\hline Sample codes & $\begin{array}{l}\text { Cross point, } \\
G^{\prime}=G^{\prime \prime}(\mathrm{Pa})\end{array}$ & $\begin{array}{l}\omega \\
(\mathrm{rad} / \mathrm{s})\end{array}$ & $\begin{array}{l}M_{w}{ }^{*} \\
(\mathrm{~g} / \mathrm{dl})\end{array}$ & $\begin{array}{l}\text { MFI } \\
(\mathrm{g} / 10 \mathrm{~min})\end{array}$ \\
\hline $\mathrm{A}$ & 157,400 & 251 & 521,000 & $8.0 \pm 0.6$ \\
$\mathrm{~B}$ & 107,700 & 398 & 412,000 & $8.6 \pm 0.3$ \\
$\mathrm{C}$ & 117,100 & 398 & 422,000 & $8.4 \pm 0.6$ \\
$\mathrm{D}$ & 137,300 & 398 & 443,000 & $9.6 \pm 0.0$ \\
\hline
\end{tabular}

*GPC measurements in a Viscotek equipment. 
Comparing the results between the compositions analyzed, a different behavior tends to occur with the composite with higher talc content (7 wt.\%). An increasing in the cross point value $\left(G^{\prime} / G^{\prime \prime}\right)$ was observed, maintaining constant the crossover frequency value. It indicates that a material with more elastic behavior tended to be obtained. A slight increasing in the $M_{w}$ value was observed.

The relaxation processes that occur during the viscoelastic transitions are influenced by the final composite morphology. The presence of particle agglomerates in polymeric system with fillers can be detected by using a representation called Cole-Cole plot. In this representation, the $\eta^{\prime \prime}$ parameter (where $\left.\eta^{\prime \prime}=G^{\prime} / \omega\right)$, called imaginary viscosity is plotted against dynamic viscosity, $\eta^{\prime}$ (where $\eta^{\prime}=G$ '”/ $\omega$ ). The plot should be a perfect arc if higher order structures are absent and the relaxation behavior of the melt can be described by a single relaxation time. A broad relaxation time spectrum leads to the flattening of the arc, a tail or an increasing correlation [12].

The dynamic viscosity $(\eta$ ') of the pure PLA and PLA/talc composites is plotted in this way in Fig. 4.

The Cole-Cole plot is arc like for PLA and its composites, indicating the absence of filler aggregates. It can be seen that the addition of talc leads to an increase of the viscous behavior of the composites. However, as talc content increased, a decreasing of this behavior was observed, and materials with higher elastic behavior tended to be produced.

It's important to say that rheology is a very sensitive tool to detect aggregation, but further analysis should be used to confirm the presence of higher order structures, such as scanning electron microscopy (SEM).

\subsection{Morphological Analysis}

The fracture surfaces were studied by SEM. According to Jiang et al. [13], in the fracture processes of thermoplastic polymer materials, yield crazing or shear yielding occurs. As seen in Fig. 5, the fracture

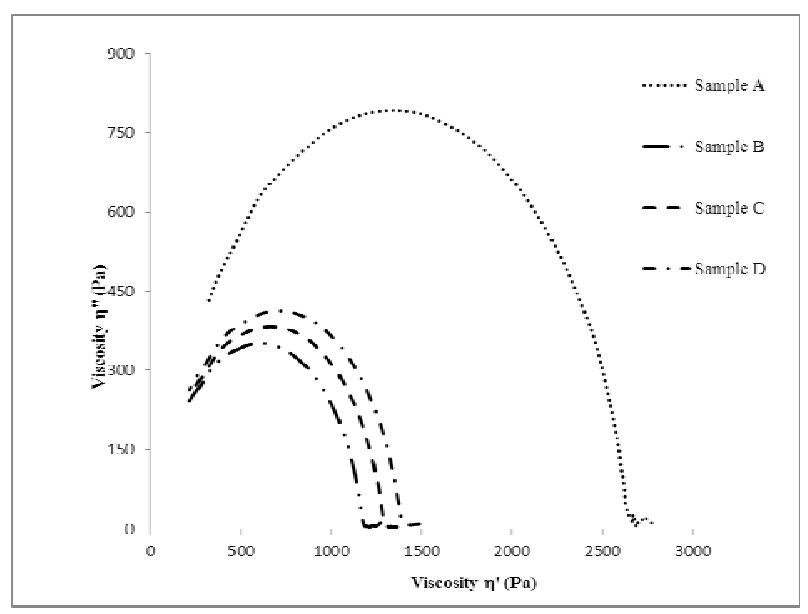

Fig. 4 Cole-Cole representation of PLA and PLA/talc melts.

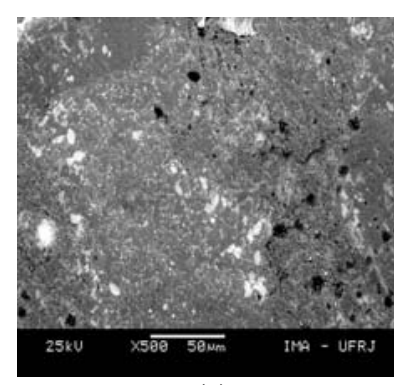

(a)

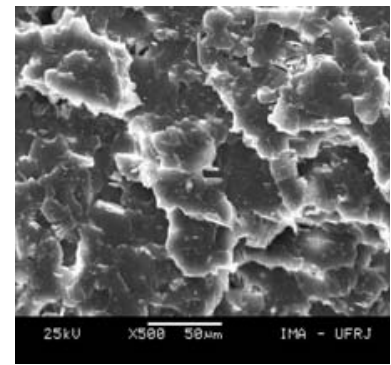

(c)

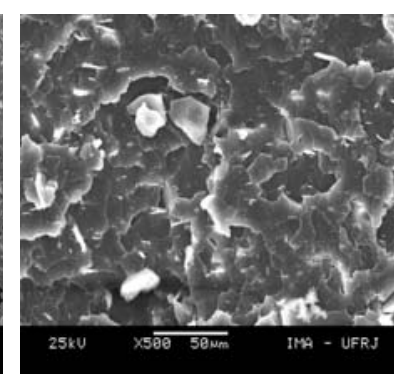

(b)

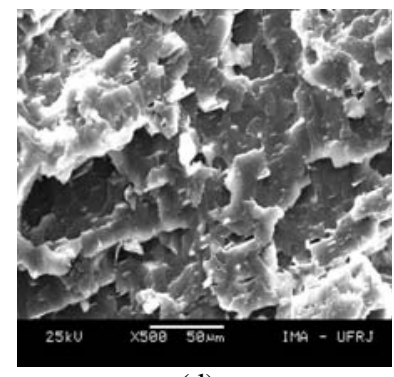

(d)
Fig. 5 SEM micrographs of PLA and PLA composites. (a) Sample A; (b) Sample B; (c) Sample C and (d) Sample D.

surface of PLA appears more brittle since less plastic deformation can be detected on the fracture surface of the pure polymer. When talc is added to the PLA matrix, the fracture surface of PLA/talc showed characteristics of plastic deformation caused by more crazes, that appeared as talc content is increased. Even in the composition with more talc content (Sample D), no particle agglomeration appeared, indicating that a good dispersion of the filler on the melt was achieved. These results confirm the rheology data obtained. 


\section{Conclusions}

The rheological behavior showed that the addition of talc in the PLA matrix led to produce materials with lower viscosity values, indicating that the mineral filler, in the composition analyzed, acted as a lubricant; but, as the talc content is increased, a tendency to produce materials with higher viscosity values was observed. The Cole-Cole representation showed that can be an useful tool to identify the presence of aggregates. The results were confirmed with SEM analysis.

\section{Acknowledgments}

The authors thank the financial support by CAPES and MAGNESITA COMPANY to supply the filler, talc.

\section{References}

[1] C.W. Zhou, J.X. Zhao, Q.L.B. Meng, Z.H. Wu, Study on mechanical, termal properties and morphology of biodegradable poly(butylene succinate)/nano- $\mathrm{TiO}_{2}$ composites, Intern. Polymer Processing XXVIII 5 (2013) 483-488.

[2] Z. Gui, C. Lu, S. Cheng, Comparison of the effects of commercial nucleation agents on the crystallization and melting behavior of polylactide, Polymer Testing 32 (2013) 15-21.

[3] K. Hamad, M. Kassem, F. Deri, Rheological and mechanical properties of poly(lactic acid)/polystyrene polymer blend, Polymer Bull 65 (2010) 509-519.

[4] T. Fujimaki, Processability and properties of aliphatic polyesters, "BIONOLLE”, synthesized by polycondensation reaction, Polym. Degrad. Stab. 59 (1998) 209-214.

[5] T. Uesaka, K. Nakane, S. Maeda, T. Ogihara, N. Ogata, Structure and physical properties of poly(butylene succinate)/cellulose acetate blends, Polymer 41 (2000) 8449-8454.

[6] M. Jamshidian, E.A. Tehrany, M. Imran, M. Jacquot, S. Desobry, Poly-lactic acid: Production, applications, and release studies, Comprehensive Reviews in Food Science and Food Safety 9 (2010) 552-571.

[7] S.K. Kang, S.I. Lee, T.J. Lee, R. Narayan, B.Y. Shin, Effects of biobased and biodegradable nucleating agent on the isothermal crystallization of poly(lactic acid), Korean J. Chem. Eng. 25 (2008) 599-608.

[8] D.H.S. Souza, C.T. Andrade, M.L. Dias, Rheological behavior of poly(lactic acid)/synthetic mica nanocomposites, Materials Science and Engineering C 33 (2013) 1795-1799.

[9] R. Wagener, T.J.G. Reisinger, A rheological method to compare the degree of exfoliation of nanocomposites, Polymer 44 (2003) 7513-7518.

[10] M.T. Martyn, P.D. Coates, Pressure variation during interfacial instability the coextrusion of low density polyethylene melts, Intern. Polymer Processing XXVIII 5 (2013) 516-527.

[11] R.E.S. Bretas, M.A. D’Avila, Melt Polymer Rheology, in: UFSCar Press, São Carlos/SP, Brazil, 2000, pp. 137-153.

[12] A. Kiss, E. Fekete, B. Pukánszky, Aggregation of $\mathrm{CaCO}_{3}$ particles in PP composites: Effect of surface coating, Composites Science and Technology $67 \quad$ (2007) 1574-1583.

[13] L. Jiang, J. Zhang, M.P. Wolcott, Comparison of polylactide/nano-sized calcium carbonate and polylactide/montmorillonite mechanisms, Polymer 48 (2007) 7632-7644. 